\title{
ANTIHYPERLIPIDEMIC ACTIVITY OF COMMIPHORA MUKUL AGAINST ATHEROGENIC DIET-INDUCED HYPERLIPIDEMIA IN EXPERIMENTAL RATS
}

\author{
JAFFAR SHAIK ${ }^{1 *}$, ZEENATH KHAN ${ }^{2}$ \\ ${ }^{1}$ Department of Biochemistry, Acharya Nagarjuna University, Guntur, Andhra Pradesh, India. ${ }^{2}$ Department of Sciences, Prince Sultan \\ Military College for Health Sciences, Dammam, KSA. Email: shaikjaffar2008@yahoo.com
}

Received: 17 January 2017, Revised and Accepted: 07 March 2018

\begin{abstract}
Objective: The aim of the present study was to investigate the effect of antihyperlipidemic activity of ethyl acetate extract of Commiphora mukul (EECM) gum resin and compares its efficacy with that of atorvastatin against atherogenic diet-induced hyperlipidemia model using rat as an experimental animal.
\end{abstract}

Methods: Wistar albino rats were divided into five groups. Control animals received normal diet, hyperlipidemic control; rats treated with EECM 100 and $200 \mathrm{mg} / \mathrm{kg}$ and positive control, receiving standard drug atorvastatin $2.70 \mathrm{mg} / \mathrm{kg}$.

Results: Increase the level of serum cholesterol, phospholipids, high-density lipoproteins, low-density lipoproteins, and triglycerides causing hyperlipidemia further leading to the development of atherosclerosis. On the other hand, oral administration of ethyl acetate extract of $C$. mukul at dose of $200 \mathrm{mg} / \mathrm{kg}$ for 15 days resulted in the prevention of above abnormalities.

Conclusion: The results suggest that EECM could be beneficial in the treatment of atherosclerosis, characterized by atherogenic lipoprotein profile and abnormalities in lipid metabolism.

Keywords: Commiphora mukul, Antihyperlipidemic, High-density lipoproteins, Atorvastatin.

(C) 2018 The Authors. Published by Innovare Academic Sciences Pvt Ltd. This is an open access article under the CC BY license (http://creativecommons. org/licenses/by/4. 0/) DOI: http://dx.doi.org/10.22159/ajpcr.2018.v11i6.24800

\section{INTRODUCTION}

Hyperlipidemia is a heterogeneous disorder commonly characterized by elevated serum total cholesterol, low-density and very low-density lipoprotein cholesterol (VLDL-C), triglycerides (TG), and decreased high density lipoprotein (HDL) levels [1]. Hyperlipidemia is one of the greatest risk factors contributing to the prevalence and severity of atherosclerosis and subsequent coronary heart disease [2]. Liver synthesizes two-third of the total cholesterol made in the body. The rate-limiting enzyme is 3-hydroxy-3-methylglutaryl (HMG$\mathrm{CoA}$ ) reductase and provides feedback regulation by controlling the cholesterol concentrations in cells. Treatment of hyperlipidemia involves diet control, exercise, and the use of lipid-lowering diets and drugs [3]. The most commonly employed drugs for the treatment of hyperlipidemia include HMG-CoA reductase inhibitors, also called as statins. The World Health Organization (WHO) estimates that high blood cholesterol contributes to approximately $56 \%$ cases of cardiovascular diseases worldwide and causes about 4.4 million deaths each year. Hyperlipidemia was characterized by increase in LDL-C, VLDL, and decrease in HDL-C serum levels.

Current projections suggest that by the year 2020, India will have the largest CVD burden in the world. One of the major initiating events in atherosclerosis is oxidative damage to the cholesterol component of the LDL, known as LDL oxidation, which forms atheromatous plaque progressing to CVD. The WHO estimates that $80 \%$ of the people of developing countries rely on traditional medicines, mostly plantderived drugs, for their primary health needs. India is well known for its rich traditional systems of medicine, that is, Ayurveda, Siddha, and Unani besides a vast reservoir of living traditions of ethnomedicine. Several modern drugs in use such as statins, fibrates, nicotinic acid and resins [3], lower blood cholesterol level, either by inhibiting endogenous synthesis and/or by lowering cholesterol absorption from the intestine [4]. Due to their side effects, people are looking for safer alternatives, and the search for new drugs capable of reducing and regulating serum cholesterol level has gained interest resulting in numerous reports on significant activities of natural agents.

The herbal product used in the current study is Commiphora mukul. It is used as a traditional medicine for the treatment of lipid disorders. This work elucidates the efficacy of $C$. mukul in the treatment of hyperlipidemia induced with atherogenic diet. It belongs to family Burseraceae, commonly known as Guggul, is found in western India and has been used as a valued herb in Ayurveda medicine for over 2500 years. Traditional uses of $C$. mukul are for its anti-inflammatory, antispasmodic, carminative, astringent, sedative, stomachic, diuretic, antidiabetic, and antihyperlipidemic [5]. The chemical composition of $C$. mukul is a very complex and has not been well defined. The chemical composition of $C$. mukul is very complex and has not been well defined. It contains sugars (sucrose, fructose), amino acids, camphorene, cembrene, allylcembrol, resin, oil, and several steroids. Only some steroid components have been purified including $\mathrm{Z}$ and E guggulsterones, which have been shown to be responsible for hypolipidemic activity [6]. Guggulsterones inhibit cholesterol synthesis in the liver through antagonism of the farnesoid $X$ receptor and the bile acid receptor [7].

The present study is to investigate the $C$. mukul has protective effect on plasma lipid profile in the treatment of hyperlipidemia induced with atherogenic diet.

\section{METHODS}

Chemicals

Atorvastatin obtained from local pharmacy, Hyderabad (Dr. Reddy's Laboratories, Hyderabad). Diagnostic kits for estimation were 
purchased from Merck Diagnostics India Ltd. anesthetic ether, ethyl acetate, and ethanol (SD Fine Chemicals, Mumbai).

\section{Atherogenic diet}

Experimental hyperlipidemic diet: Experimental diet consists of wellpulverized mixture of cholesterol $-400 \mathrm{mg} / \mathrm{kg}$, cholic acid $-50 \mathrm{mg} / \mathrm{kg}$, and coconut oil. This mixture is made into paste-like molds and is fed to the rats.

\section{Plant material}

The Guggul gum resin was collected from local market in Vijayawada (Chemiloids, manufacturers, and exporters) and was authenticated. The collected gum resin was dried and powdered to a coarse consistency. The powder was passed through 40 \# mesh particle size and stored in airtight container at room temperature.

\section{Preparation of plant extract}

About $38 \mathrm{~g}$ of dried and finely ground C. mukul resin was taken in a $500 \mathrm{~mL}$ round-bottomed flask and extracted under stirring with $175 \mathrm{~mL}$ of ethyl acetate solution first at room temperature, then under mild reflux of $3 \mathrm{~h}$ using condenser. After filtering the insoluble matter, the residue was extracted further with $175 \mathrm{~mL}$ of the same solvent under the same conditions. The collected extracts were treated with $0.02 \mathrm{~g}$ of charcoal and after $3 \mathrm{~h}$ of mild reflux, filtered. A bright yellow clear solution was obtained. This solution was concentrated at 40 to a very thick honey pasty form and the residue was diluted with $750 \mathrm{~mL}$ of ethanol. The ethanolic solution was filtered and concentrated to obtain amber colored semifluid material. Ethyl acetate extract of $C$. mukul (EECM) was stored in an airtight container.

\section{Treatment with atherogenic diet}

The prepared atherogenic diet was used in place of normal pellet diet to all the groups except control. Rats were exposed to atherogenic diet and water ad libitum for 20 days and were used to study the effect of EECM against experimental hyperlipidemia.

\section{Preparation of test and standard for administration}

EECM was weighed and suspended in $2 \%$ tween solution to attain the required doses. Groups 2 and 3 were treated with EECM 100 and $200 \mathrm{mg} / \mathrm{kg}$, respectively, for 15 days. Group 4 rats were treated with atorvastatin $2.7 \mathrm{mg} / \mathrm{kg}$ using oral feeding needle.

\section{Selection of animals}

On the last day of treatment, rats were selected for jumping based on their lipid profile. Rats which have shown an increased LDL-C were selected for the study.

\section{Experimental animals}

Adult albino rats of Wistar strain (150-250 g) of either sex were procured from animal house of Bengaluru. The animals are maintained at normal temperature in $12 \mathrm{~h}$ light and $12 \mathrm{~h}$ dark cycles. The animals were provided food and water ad libitum. The protocol was submitted to IAEC Committee before the initiation of the study. The animals are divided into five groups each containing three animals.

\section{Experimental design}

In the present experiment, control animals received normal diet; hyperlipidemic control rats received atherogenic diet, atherogenictreated group and normal-treated group received an ethanolic leaf extract of the gum resin of $C$. mukul by orogastric tube at dose of 100 and $200 \mathrm{mg} / \mathrm{kg}$ body weight for 15 days. Based on preliminary experiment on dose-dependent antihyperlipidemic effect of CMEEt, a dose $<200 \mathrm{mg} / \mathrm{kg}$ body weight was not expected to be effective in rats.

\section{Collection of blood sample}

Blood was collected by retro-orbital puncture, under mild ether anesthesia on the last day of treatment and subjected to biochemical analysis for estimating TG, HDL-C, LDL-C, and total cholesterol using various estimations.

\section{Biochemical analysis}

The biochemical parameters (HDL-C, LDL-C, total cholesterol, and TG) have been investigated in serum once a week during the treatment with ethyl acetate extract of the plant and atorvastatin preparations. Blood was withdrawn using heparinized capillaries from the retro-orbital sinus in the overnight-fasted animals. The serum was obtained after centrifuging the blood, which was used to estimate the concentration of biochemical parameters using the semi-autoanalyzer and relevant lipid profile kits.

The serum samples were analyzed for total cholesterol, TG, and HDL-C and LDL-C using standard protocol method (Span diagnostic Ltd., Surat, India).

\section{Statistical analysis}

The results of the study were expressed as mean \pm SEM. Data were analyzed using one-way analysis of variance test followed by Dunnett's test. Values with $\mathrm{p}<0.05$ were considered as statistically significant.

\section{Effect of EECM on serum total cholesterol}

A significant increase in the serum cholesterol levels was observed in rats treated with atherogenic diet when compared with that of normal control. Treatment with EECM $100 \mathrm{mg} / \mathrm{kg}$ did not show any significant decrease in the total cholesterol levels (Table 1). Whereas, treatment with EECM $200 \mathrm{mg} / \mathrm{kg}$ had shown a statistically significant $(\mathrm{p}<0.01)$ decrease in total cholesterol levels.

\section{Effect of EECM on serum TG}

Rats treated with atherogenic diet had shown a statistically significant $(\mathrm{p}<0.001)$ increase in the serum TG levels. Treatment with EECM $100 \mathrm{mg} / \mathrm{kg}$ had shown a marked $(\mathrm{p}<0.05)$ decrease in the serum TG levels. Whereas, treatment with EECM $200 \mathrm{mg} / \mathrm{kg}$ had shown a statistically significant $(\mathrm{p}<0.01)$ decrease in TG levels. A statistically significant $(p<0.001)$ decrease in the TG levels was observed in rat treated with atorvastatin (Table 1 )

\section{Effect of EECM on serum HDL-C levels}

Rats of negative control group had shown a statistically significant $(p<0.01)$ decrease in the HDL-C levels when compared with that of control group. Whereas, rats treated with EECM 100 and $200 \mathrm{mg} / \mathrm{kg}$ had shown a marked increase $(\mathrm{p}<0.01)$ in the HDL-C levels when compared with that of atorvastatin-treated animals (Table 1).

\section{Effect EECM on serum LDL-C levels}

A significant increase in the LDL-C levels was observed in negative control group. Whereas, treatment with EECM 100 and $200 \mathrm{mg} / \mathrm{kg}$ had shown a statistically significant $(\mathrm{p}<0.001)$ decrease in the serum LDL-C levels. Moreover, treatment with atorvastatin had shown a statistically significant $(p<0.001)$ decrease in the LDL-C levels (Table 1$)$.

\section{DISCUSSION}

The present study was carried out to examine the antihyperlipidemic effect of ethanolic leaf extract of $C$. mukul gum resin on atherogenicinduced hyperlipidemia rats in dose-dependent manner. Treatment with atherogenic diet elevates the level of serum cholesterol, phospholipids, HDL, LDL, and TG causing hyperlipidemia further leading to the development of atherosclerosis. Both cholesterol and phospholipids were reduced remarkably on treatment with ethanolic extract of gum resin of $C$. mukul. This lipid-lowering effect may be due to the inhibition of hepatic cholesterogenesis and catabolic conversion of cholesterol to bile acids in the liver. Our findings were similar with that of earlier studies; Satyavati [9] studied the effect of gum guggul on the lipid levels of hyperlipidemic rabbits. Following her studies, a wide range of efforts has confirmed the hypolipidemic effect of gum guggul and leads to the identification of the compounds such as E- and Z-guggulsterone the isomers of gum guggul as the active hypolipidemic agents $[8,9]$.

Several mechanisms have been proposed for the effects of guggul. It may decrease hepatic steroid production, ultimately increasing 
Table 1: Effect of $C$. mukul ethanolic leaf extract gum resin intragastric treatment for $\mathbf{1 5}$ days on plasma lipid profiles in cholesterol-induced hyperlipidemia

\begin{tabular}{|c|c|c|c|c|c|}
\hline S.No & Group & TG & Total-cholesterol & LDL-C & HDL-C \\
\hline I & Normal control & $129.5 \pm 3.98$ & $70 \pm 1.73$ & $74 \pm 2.74$ & $49 \pm 3.14$ \\
\hline II & Hyperlipidemic control & $182.5 \pm 4.04^{*}$ & $100.75 \pm 6.63^{*}$ & $139 \pm 1.73^{*}$ & $30.5 \pm 1.15^{*}$ \\
\hline III & EECM (100 mg/kg) & $158.25 \pm 3.89 * *$ & $80.5 \pm 2.88^{* *}$ & $113.25 \pm 4.33^{* *}$ & $38.5 \pm 4.2 * *$ \\
\hline IV & EECM (200 mg/kg) & $136 \pm 3.17^{* *}$ & $73 \pm 4.61^{* *}$ & $85.25 \pm 2.02^{* *}$ & $50.75 \pm 1.09^{* *}$ \\
\hline V & Extract (2.7 mg/kg) & $128.44 \pm 5.4^{* *}$ & $65 \pm 3.46^{* *}$ & $74 \pm 1.15^{* *}$ & $52.75 \pm 2.02^{* *}$ \\
\hline
\end{tabular}

the catabolism of plasma LDL-C. Alternatively, the proposed active components of guggul, guggulsterones $\mathrm{E}$ and $\mathrm{Z}$, may increase hepatic binding sites for LDL-C, thus increasing LDL-C clearance. Still, another possibility is the prevention of cholesterol synthesis in the liver by ketogenic steroids [8,10]. Guggulsterones $\mathrm{E}$ and $\mathrm{Z}$ are effective antagonists of the bile acids receptor, farnesoid $\mathrm{X}$ receptor, which is primarily expressed in the liver, kidney, and small intestine; it regulates the expression of genes involved in cholesterol/bile acids homeostasis, allowing more cholesterol catabolism and excretion from the body [10]. Studies of the efficacy of guggul for hypercholesterolemia have produced conflicting results and information currently available indicates that guggul may be effective for lowering total cholesterol and TG in patients on a non-Western diet [11].

Statins competitively inhibit conversion of HMG-CoA to mevalonate (rate-limiting step in cholesterol synthesis) by the enzyme HMG-CoA reductase [11]. This results in compensatory increase in LDL receptormediated uptake and catabolism of IDL and LDL. Over long term, feedback induction if HMG-CoA reductase tends to increase cholesterol synthesis, but a steady state is attained with a dose-dependent lowering of LDL-C levels [8]. The drugs statins decrease the LDL-Cholesterol by $20-55 \%$, increase the HDL-C levels by $5-15 \%$ and decrease the Triglycerides by $10-35 \%$.

Ethanol extract of nutshell (500 mg/kg) decreased serum LDL-C and very VLDL-C levels on 60 days of treatment in atherogenic male rabbits. Cholesterol mobilization from liver and prevention of deposition in peripheral tissues were observed and the inhibition of cholesterol absorption at intestine may be considered as the possible mode of action [12]. Ethanolic extract of bark of Terminalia arjuna (100 and $500 \mathrm{mg} /$ $\mathrm{kg}$ ) treated to atherogenic albino rabbits reduced TC after 60 days. Both the dosage forms were observed effective in decreasing TC and LDL-C levels, whereas the TC:HDL ratio was influenced by the higher dose [19]. In a randomized placebo-controlled trial, T. arjuna tree bark powder (500 mg) significantly decreased TC level in 105 successive CAD patients [13]. The hypolipidemic action of T. arjuna coupled with the enhancement of prostaglandin E2 such as activity, antiarrhythmic, antihypertensive, and HDL-C raising properties make it an imminently cardioprotective product for the overall management of CAD [13]. The lipid lowering activity (LLA) of Phyllanthus niruri has been studied in triton and cholesterol fed hyperlipemic rats. Serum lipids were lowered by $P$. niruri extract orally fed ( $250 \mathrm{mg} / \mathrm{kg}$ b.w.) to the triton WR-1339 induced hyperlipemic rats. Chronic feeding of this drugs $(100 \mathrm{mg} / \mathrm{kg}$ b.w.) in animals simultaneously fed with cholesterol $(25 \mathrm{mg} / \mathrm{kg}$ b.w.) for 30 days caused lowering in the lipids and apoprotein levels of VLDL and LDL in experimental animals [14]. The antihypercholesterolemic effect of this plant was assessed to be mediated through the inhibition of hepatic cholesterol biosynthesis, increased excretion of bile acids, and enhanced plasma LCAT activity. Fenugreek powder $(15 \%, 30 \%$, and $60 \%$ ) mixed with hypercholesterolemia inducing diet and fed to rats for 4 weeks showed decrease in serum LDL-C and VLDL-C levels. In another study, fenugreek seed powder (5\%,10\%, and 15\%) showed antilithogenic effect in mic $[14,12]$.

The fruits of Moringa oleifera $(200 \mathrm{mg} / \mathrm{kg}$ ) given along with standard laboratory diet and hypercholesterolemic diet to rabbits for 120 days were found to lower the serum cholesterol, phospholipid, TG, VLDL-C, LDL-C, and atherogenic index but were found to increase the HDL-C as compared to the corresponding control groups [15].

The hypocholesterolemic effect of sesame seed powder administered (5\% and $10 \%$ ) to hypercholesterolemic male albino rats showed decline in plasma LDL-C level, increased in hepatic HMG-CoA reductase activity, and enhanced fecal bile acids and cholesterol excretion. The hypocholesterolemic effect appeared to be due to its fiber, sterol, polyphenol, and flavonoid contents [15-17].

Flavonoids from Emblica officinalis and Mangifera indica effectively reduce lipid levels in serum and tissues of rats induced hyperlipidemia. Hepatic HMG CoA reductase activity was significantly inhibited in rats fed E. officinalis flavonoids. But increase of this enzyme was observed in rats administered $M$. indica flavonoids. LCAT showed elevated levels in rats fed flavonoids from E. officinalis and M. Indica [18].

Increased concentration of LDL-C was observed in the serum of high-fattreated rats when compared with the control. Treatment with $C$. mukul extract reduced LDL-C levels significantly. HDL-C is synthesized mainly in the intestine and liver. It has high phospholipid content and is involved in reverse cholesterol transport. HDL is considered to be a beneficial lipoprotein as it has an inhibitory effect in the $C$. mukul pathogenesis of atherosclerosis. HDL concentration was significantly increased on C. mukul extract treatment in this present investigation. The observed hypolipidemic activity of EECM may be due to the presence of plant sterols (guggulsterone E and guggulsterone) [8-11,19].

\section{CONCLUSION}

EECM had shown asignificant reduction in the serum TG, totalcholesterol, and LDL-C and a significant increase in the HDL-C, thus indicating the potential use of this extract in the treatment of hyperlipidemia and in other associated disorders such as atherosclerosis. In the light of these beneficial effects of ethyl acetate extract of $C$. mukul are especially promising in preventing lifestyle disease of the cardiovascular system. The effect on development of resistance on prolonged use is to be done in the future to explore the complete therapeutic potential of $C$. mukul.

\section{ACKNOWLEDGMENT}

We thank head of the department of biochemistry for providing facilities to carry out this research work.

\section{CONFLICT OF INTEREST}

We have no conflict of interest.

\section{REFERENCES}

1. Ballantyne CM. Clinical trial endpoints: Angiograms, events, and plaque instability. Am J Cardiol 1998;82:5M-11M.

2. Durrington PN. Diabetic dyslipidaemia. Baillieres Best Pract Res Clin Endocrinol Metab 1999;13:265-78.

3. Satoskar RS, Bhandarkar SD, Ainapure SS. Hypolipidemic drugs. In: Pharmacology and Pharmacotherapeutics. $18^{\text {th }}$ ed. Mumbai, India: Popular Prakashan; 2003. p. 567-75.

4. Sedaghat A, Samuel P, Crouse JR, Ahrens EH Jr. Effects of neomycin on absorption, synthesis, and/or flux of cholesterol in man. J Clin Invest 1975;55:12-21

5. Szekanecz Z, Koch AE, Kunkel SL, Strieter RM. Cytokines in rheumatoid arthritis. Potential targets for pharmacological intervention. 
Drugs Aging 1998;12:377-90.

6. Urizar NL, Moore DD. Gugulipid: Natural cholesterol-lowering agent. Annu Rev Nutr 2003;23:303-13.

7. Nagarajan M, Waszkuc TW, Sun J. Simultaneous determination of Eand Zguggulsterones in dietary supplements containing Commiphora mukul extract (guggulipid) by liquid chromatography. J AOAC Int 2001;84:24-8

8. Wang X, Greilberger J, Ledinski G, Kager G, Paigen B, Jurgens G. The hypolipidemic natural product Commiphora mukul and its component guggulsterone inhibit oxidative modification of LDL. Atherosclerosis 2004;172:239-46.

9. Satyavati GV. Gum guggulu (Commiphora mukul)-The success story of an ancient insight leading to a modern discovery. Indian J Med Res 1988;87:327-35.

10. Burris TP, Montrose C, Houck KA, Osborne HE, Bocchinfuso WP, Yaden BC, et al. The hypolipidemic natural product guggulsterone is a promiscuous steroid receptor ligand. Mol Pharmacol 2005;67:948-54.

11. Chander R, Khanna AK, Pratap R. Anti hyperlipidemic activity of guggulsterone, the active principle of guggulipid from Commiphora mukul. J Med Arom Plant Sci 2003;24:370-4

12. Sharma A, Mathur R, Dixit VP. Hypocholesterolemic activity of nut shell extract of Semecarpus anacardium (Bhilawa) in cholesterol fed rabbits. Indian J Exp Biol 1995;33:444-8.

13. Ram A, Lauria P, Gupta R, Kumar P, Sharma VN. Hypocholesterolaemic effects of Terminalia arjuna tree bark. JEthnopharmacol 1997:55:165-9.

14. Khanna AK, Rizvi F, Chander R. Lipid lowering activity of Phyllanthus niruri in hyperlipemic rats. J Ethnopharmacol 2002;82:19-22.

15. Ghasi S, Nwobodo E, Ofili JO. Hypocholesterolemic effects of crudemextract of leaf of Moringa oleifera Lam in high-fat diet fed wistar rats. J Ethnopharmacol 2000;69:21-5.

16. Andallu B, Radhika B. Hypoglycemic, diuretic and hypocholesterolemic effect of winter cherry (Withania somnifera, dunal) root. Indian J Exp Biol 2000;38:607-9.

17. Gupta RS, Dixit VP, Dobhal MP. Hypocholesterolaemic effect of the oleoresin of Capsicum annum L. In gerbils (Meriones hurrianae jerdon). Phytother Res 2002;16:273-5.

18. Anila L, Vijayalakshmi NR. Flavonoids from emblica officinalis and mangifera indica-effectiveness for dyslipidemia. J Ethnopharmacol $2002 ; 79: 81-7$

19. Singh RB, Niaz MA, Ghosh S. Hypolipidemic and antioxidant effects of Commiphora mukul as an adjunct to dietary therapy in patients with hypercholesterolemia. Cardiovasc Drugs Ther 1994;8:659-64. 